\title{
Ochoa syndrome
}

INSERM

\section{Source}

INSERM. (1999). Orphanet: an online rare disease and orphan drug data base. Ochoa

syndrome. ORPHA:2704

Ochoa syndrome is characterized by the association of severe voiding dysfunction and a characteristic facial expression. 